\title{
La muerte en las ciencias sociales: una aproximación
}

\author{
Sandra Gayol* y Gabriel Kessler**
}

\section{RESUMEN}

El artículo presenta las ideas centrales de los trabajos y debates que en torno a la muerte marcaron a la historia, la antropología y la sociología en el último siglo. Distingue especialmente tres momentos clave en la producción de conocimiento sobre la muerte: inicios del siglo XX, las décadas de 1960, 1970, y a partir de los ańos noventa. El artículo recorre cada uno de sus momentos, presenta las preocupaciones, el contexto que configura y propicia la emergencia de estudios, así como los hallazgos más novedosos y útiles para pensar el tema en la actualidad. Focaliza sobre todo en el tratamiento de los rituales, el cuerpo, el sentido social de la muerte y el proceso de morir. El recorrido es esencialmente a través de la bibliografía de los países centrales y, al mismo tiempo, es sensible a los aportes que podría brindar una mirada más descentrada y desde el sur. En el balance final se plantean algunas preguntas y se reformulan preocupaciones 'clásicas' a la luz de algunos datos de los países latinoamericanos.

Palabras clave

Muerte $\bullet$ cuerpo $\bullet$ proceso de morir $\bullet$ rituales $\bullet$ emociones

Death in the Social Sciences: An Approach

\section{ABSTRACT}

This article presents the central ideas of the work and debates around death that marked history, anthropology and sociology during the last century. It especially distinguishes three key moments in the production of knowledge about death:

* Doctora en Historia EHESS, París. Profesora asociada con dedicación exclusiva Universidad Nacional de General Sarmiento (UNGS) e investigadora independiente Consejo Nacional de Investigaciones Científicas y Técnicas (Conicet), Buenos Aires. E-mail: sandra.gayol@gmail.com.

** Doctor en Sociología EHESS, París. Investigador independiente Consejo Nacional de Investigaciones Científicas y Técnicas (Conicet). Profesor titular Universidad Nacional de La Plata, Buenos Aires. E-mail: gabriel_kessler@yahoo.com.

Este artículo se publica en el marco de cooperación internacional del proyecto Fondecyt $\mathrm{n}^{\circ} 1095083$. 
the early twentieth century, the 60s and 70s and the period beginning during the 90s. The article covers each of its moments, presents the concerns, the context that shapes and promotes the emergence of studies and the most novel and useful findings used to ponder about this issue. It focuses mainly on the treatment of rituals, the body, the social meaning of death and the process of dying. The route taken is essentially through the bibliography of the central countries and, at the same time, is sensitive to the contributions that could be provided by a more off-center look stemming from the south. In the final balance some questions are raised and 'classic' concerns are reformulated in the light of information that has originated in Latin American countries.

Keywords

Death $\bullet$ body $\bullet$ dying process $\bullet$ rituals $\bullet$ emotions

\section{Introducción}

A diferencia del lugar central que ha ocupado en las artes, las ciencias de la vida y la religión, la muerte ha suscitado hasta no hace mucho tiempo un interés más bien marginal en las ciencias sociales. No deja de sorprender la asimetría entre la honda y perenne preocupación que la muerte concita en la vida social de los hombres y mujeres con el reducido tratamiento dispensado por las disciplinas que de ella se ocupa. En rigor, sin haber sido un tema central, visto retrospectivamente pueden determinarse en el último siglo algunos períodos de mayor interés por el tema, en particular por la muerte del otro en otras culturas. Tanto es así, que, pocos ańos atrás, A. Robben (2004) cuestionaba a la antropología por no atreverse a tocar la muerte en las sociedades occidentales, confinando su interés por el tema sólo cuando se estudiaban sociedades remotas. Algo similar podría decirse de las otras disciplinas; sin embargo, este tabú, desinterés o dificultad para pensar aquello que se situaba del otro lado de una frontera imaginada de la vida social, hoy ha cambiado y en unas ciencias sociales crecientemente especializadas, los estudios por el tema han conocido una notable productividad.

El objetivo de este artículo es presentar las ideas centrales de los trabajos y debates que fueron marcando a la historia, la antropología y la sociología en el último siglo. No nos centraremos en todos los textos, tarea imposible debido a la cantidad de títulos existentes, sino que abordaremos los principales debates y aportes en una periodización en tres momentos en los cuales se presenta una mayor cantidad de trabajos y aproximaciones novedosas. Más focalizado en la producción conceptual 
de los países centrales que en los nuestros, no se desconoce aquí la necesidad de una aproximación propia de los países del Sur. Los tópicos principales serán los rituales, el cuerpo, el sentido social de la muerte y el proceso de morir, dejando de lado otros temas, sin duda de interés capital, como la relación entre muerte y política, las muertes violentas o el suicidio que, desde E. Durkheim, ha sido un campo de preocupación en sí mismo.

El artículo recorre cada uno de los tres períodos, presenta las preocupaciones centrales, el contexto social y político, así como los hallazgos más novedosos, útiles para pensar el tema en la actualidad. Concluye con un balance general de los trabajos analizados, dejando planteadas algunas preguntas abiertas para análisis locales.

\section{La lenta conformación de un campo de estudios}

Los comienzos de constitución de un campo de estudios sobre la muerte provienen de la antropología y la sociología, y vienen de la mano de autores que se convertirán en clásicos (Durkheim, Van Gennep, Hertz, Mauss, Malinovsky, Radcliffe Brown), en muchos casos 'padres fundadores' de ambas disciplinas. El esfuerzo de construcción disciplinar, para la antropología, y la vocación de crear una escuela, para la sociología, marcaron profundamente estos trabajos pioneros. La intención de demarcar temas, problemas, espacios específicos de indagación y metodologías fue a la par de dos preocupaciones esenciales: definir y clasificar para ordenar y hacer inteligible el espacio social y, en clave positivista, encontrar leyes generales de la sociedad a partir de la observación empírica y no por medio de especulaciones metafísicas.

Los estudios desembocan en la muerte a partir de un interés primordial por la diversidad de expresiones religiosas. La muerte es, entonces, el punto de llegada que permite describir y explicar la violenta y compleja explosión de manifestaciones religiosas, pues son estas las que dan cuerpo y forma a la salvadora y confortable creencia en la inmortalidad del espíritu independientemente del cuerpo (Malinowski 1948). El carácter universal e inevitable de la muerte biológica se aísla, así, para ver cómo se comportan y cuál es la respuesta de las diferentes culturas frente a este hecho inevitable. No es la muerte mía, la muerte tuya o la muerte nuestra, sino la del otro que acontece en otra cultura. Esta doble alteridad diseńa el vínculo muerte/creencias que articula la mayoría de las reflexiones y sustenta una idea capital: la universalidad de la muerte va a la par de la universal negación de ella. La negación de la muerte, que como veremos tendrá enorme fortuna en muchos estudios realizados a partir de los años sesenta, se entiende aquí no 
como un escamoteo u ocultamiento sino como la negación del fin, del total aniquilamiento. Las religiones, a través de la fe, ofrecen la posibilidad de pensar la muerte no como un final sino como la puerta de ingreso al más allá. Esto puede adoptar, a su vez, una pluralidad de formas y los caminos para llegar a él pueden también ser disímiles: dependen siempre de la forma de morir. La dicotomía buena muerte-mala muerte es clave, pues "los hombres y las mujeres prefieren morir una muerte en lugar de otra y aunque no implicaban atributo moral alguno conferido a algunas de las formas; una cierta fascinación por morir en la batalla y el temor a la brujería y la enfermedad parecían ser, de hecho, las causas de esas predilecciones" (Malinowski 1948:142). Estas predilecciones entre los habitantes de las islas Trobiand, sabemos, no pueden ser generalizables y más tarde hemos aprendido que las formas de morir no sólo pueden ser sometidas a juicios morales, sino que varían históricamente y están históricamente situadas. Más allá de estos reajustes y redefiniciones, la dicotomía buena muerte-mala muerte fue importante en estos trabajos pioneros en tanto indicadores de formas deseables de morir, de caminos posibles para llegar al más allá y de rituales merecidos y esperables.

Como una etapa distinta que inicia un nuevo momento para el muerto y también para la sociedad, la muerte es pensada como un proceso que no provoca, por lo demás, la destrucción instantánea del individuo. La inmortalidad, vimos, es la del espíritu, mientras que es el cuerpo el que se descompone. Las ceremonias establecen estas diferencias y garantizan esta separación. Dos autores son aquí esenciales: Arnold Van Gennep (1960 [1909]) y Robert Hertz (1960 [1907]). El primero, abocado a los ritos de pasaje en general y a los funerales en particular, por cuanto son estos, sostiene, los ritos de separación más significativos. El segundo, si bien trabajó la idea de ritos de pasaje, concentró su interés en el significado social del funeral, en el papel que juega el cuerpo y las dobles exequias. En 1909, en Les rites de passages, Arnold Van Gennep estableció la relación entre creencias y prácticas mortuorias. Sostuvo que en el curso de una vida, en el desarrollo social de un individuo, este debe pasar por distintas y numerosas situaciones de crisis que provocan un cambio de estatus, de estado, de clase social y de edad. Para estas situaciones existen ritos que tienen el propósito esencial de facilitar el pasaje de una posición definida a otra posición que asimismo está bien definida. Los rituales son necesarios para exorcizar el caos, el desorden y la disrupción que pueden afectar a una sociedad en un momento dado. La función es 'domar' los peligros, evitar el desorden y garantizar la continuidad de la sociedad y la cultura. Dos años antes, en 1907, Robert Hertz escribió un artículo publicado por primera vez en L'année Sociologique, "Contribution a une répréséntation collective de la mort", en donde prestó especial importancia al caso de las dobles exequias: combinando trabajos 
de campo con etnografías realizadas por otros autores para otros lugares, observó que, en la mayoría de las culturas, al morir una persona esta era sometida a dos entierros: uno transitorio y otro definitivo. Esto se debía, según el autor, a la creencia de que una persona está verdadera y definitivamente muerta cuando su cuerpo se ha desintegrado totalmente y sólo quedan sus huesos. Precisamente, el objetivo del primer entierro es permitir que la carne se pudra, se caiga y que los huesos queden completamente secos. Esta transformación no es a los ojos de los primitivos sólo una desintegración física: cambia el carácter del cadáver, que se convierte en un nuevo cuerpo y, consecuentemente, es una condición necesaria para la salvación del alma. La muerte es una transición: a medida que progresa, renace; cuando el cuerpo termina de caer en ruinas, un nuevo cuerpo toma forma, con el cual el alma -con todos los ritos necesarios- puede entrar a la otra existencia, generalmente superior a la que tuvo hasta ese momento. Con el último entierro termina el período intermedio: el muerto ha cambiado por completo de condición y reúne su cuerpo con los de sus ancestros.

Van Gennep y Hertz plantean, casi en paralelo, la existencia de rasgos estructurales en los rituales. Más allá de la diversidad y variedad de elementos específicos que pueden mostrar, más allá de sus características particulares, todos los rituales comparten una estructura tripartita: separación, transición y reincorporación. Si la intensidad y extensión de cada uno de ellos varía, aparecen en todas las ceremonias. La función es también universal, pues "marcan la socialización de las transiciones más importantes en la vida humana -como el nacimiento, la adultez, el casamiento y la muerte- y contribuyen a preservar la estabilidad de la sociedad, regulando la posición del individuo en su seno" (Van Gennep 1960).

Los rituales incorporan al individuo en un nuevo estatus, pero también "son ceremonias restauradoras de los sentimientos morales que han sido perturbados por los cambios de la vida social del grupo" (Radcliffe-Brown 1972:201). Y están, también, socialmente prescriptos. Especialmente para Radcliffe Brown, Malinowski y Mauss, las formas de expresión del dolor - por medio de las lágrimas, gritos y distintas manifestaciones corporales- son socialmente construidas en dos sentidos: es una obligación hacia los otros sentir dolor por el prójimo y la ausencia de dolor es en muchas sociedades y circunstancias un problema; y la forma, intensidad, duración y distribución relativa de ese dolor también es prescripto socialmente de acuerdo a la relación del vivo con el difundo, y de la posición que ocupa el difunto.

Las sofisticadas etnografías que signaron estas primeras aproximaciones al tema de la muerte fueron indiferentes a la comparación (Robben 2004), escasamente atentas al problema de la generalización y son metodológicamente homogéneas: se basan en la observación participante y en el análisis cualitativo interesado por 
los valores. Pero dejan planteadas preguntas sobre el tema que la sociología, más allá del interés por el suicidio en una de sus obras iniciales, no abordará. En esta doble alteridad, la muerte en las culturas occidentales tardará en convertirse en un legítimo objeto de estudio.

\section{La muerte en un contexto de cambio}

Tal desinterés por la muerte en Occidente llevó a William Faunce y Robert Fulton (1957) a sostener que se trataba de un área de investigación descuidada por la sociología. Suscitaba su atención que no se hubiera tratado el tema durante la Segunda Guerra Mundial. Tanto es así que en The American Soldier (Stouffer et al. 1949), dirigido a los temores, la valentía, la cobardía y en general la moral de las tropas, la muerte estuvo ausente de la indagación y de las explicaciones sobre los miedos. En rigor, la excepción se hallaba en la emergente especialidad de la sociología de las profesiones, para la cual la entonces novedosa figura del 'director de funerales' (Habenstein y Lamers 1955) en la organización de las exequias era el resultado de la conjunción del proceso de secularización y de la agresiva penetración del mercado en la industria fúnebre de Estados Unidos, ya en la primera mitad del siglo XX (Mitford 1963).

En las más de dos décadas que van desde el llamado de atención de dichos autores hasta un punto de inflexión ocurrido a mediados de la década de 1980, los fundamentos de una sociología de la muerte comienzan a erigirse, sobre todo en Estados Unidos, con diversas preguntas, investigaciones empíricas y desarrollos conceptuales. El levantamiento del tabú sobre el tema se debió menos a transformaciones endógenas de las disciplinas que a cambios en el contexto médico, social y legal.

R. Fox (1981) describe este nuevo contexto: en primer lugar, el creciente control de las enfermedades infecciosas a través de desarrollos farmacológicos después de la Segunda Guerra Mundial implicó una disminución de la gravedad y mortalidad de dolencias tales como la neumonía, la tuberculosis y la septicemia, ubicándose en su lugar, tanto como causa de mortalidad como de preocupación social, las enfermedades crónico-degenerativas, en particular las cardiovasculares y el cáncer, entrando en lo que se llamó la 'sociedad posinfecciosa'. Una de sus consecuencias es el cambio en el patrón demográfico: la esperanza de vida se extiende y, con ello, la experiencia de la vejez -antes más limitada-adquiere un peso demográfico mayor. La muerte, entonces, de un evento disruptor en cualquier momento de la vida producto de las dolencias infecciosas, se ubica ahora sobre todo como desenlace de la ancianidad. De este modo, se transforma en un evento más previsible en un 
doble sentido: esperable como fin natural del ciclo vital y pasible de ser anticipado realizándose los arreglos necesarios.

Los avances de la medicina respecto de las enfermedades crónicas, en particular el cáncer, permitía quizás no la cura, pero sí predecir un tiempo de sobre vida y la necesidad de hablar con los pacientes o al menos con los familiares. Pero, además, el cáncer se erige como metáfora del mal, de la invasión, tanto en el pensamiento corriente como en la política (Sontag 1977). Los tratamientos contra el cáncer dan lugar a una serie de debates y cuestionamientos sociales. Una fue la naciente crítica al poder médico y a los modos de tratar la enfermedad. Serán médicos, pero sobre todo psicólogos, enfermeras, trabajadores sociales quienes alerten sobre la contracara de esta tecnología en expansión: la deshumanización del cuidado del paciente; un cuerpo sometido hasta sus últimos momentos a intentos de prolongar la vida, sin respetar sus deseos, sin compartir información, sin consentimiento y sin facilitar un pasaje inexorable.

Fox (1981) señala un segundo movimiento que se produce también desde el campo médico: el proceso de redefinición de los límites entre la vida y la muerte, debido a los avances no lineares de la medicina. En efecto, de un lado, innovaciones como la reanimación cardíaca se volvieron posibles pero sin que se acompañe por mayores posibilidades de reanimación cerebral. Su resultado: individuos que respiraban artificialmente pero cuya actividad cerebral había cesado. No fue una mera disyunción teórica el motor de los debates, sino las exigencias de la naciente tecnología de trasplantes de órganos, que precisaban de la extracción lo antes posible en los pacientes clínicamente muertos (Seale 2003). Cambia el estatus de la muerte, tal como se la conocía hasta entonces, dejando de ser un estado necesariamente evidente (el pulso, el aliento, el ritmo cardíaco) para ser el resultado de la evaluación de ciertos indicadores obtenidos por medios técnicos. Si bien tal criterio se fue extendiendo a otros países, no dejó de causar rechazos en ciertas culturas, como en el Japón. Por último, la muerte empieza a estar mucho más presente en la naciente televisión, en películas, en series.

\section{Las ciencias sociales del período frente a la muerte}

¿Qué sucede con las ciencias sociales en este contexto? Se hacen eco de la demanda de nuevos guiones culturales para poder manejar y dar sentido a este nuevo lugar de la muerte. Una primera vertiente intentó sobre todo describir y prescribir el proceso de morir. La obra más significativa fue $O n$ Death and Dying de la psiquiatra suiza Elisabeth Kübler-Ross (1969), a partir de su experiencia profesional 
con doscientos casos de enfermos incurables. El trabajo señala una serie de cinco fases en el proceso de morir: negación, angustia, negociación/regateo, depresión y aceptación (Riley 1983). Las cinco fases, criticadas por ser más prescriptivas que descriptivas, imponiendo un modelo de agonía 'normal', son tomadas en la formación de las profesiones y también por los familiares que tienen que hacer frente a una nueva configuración del proceso de morir.

En paralelo surge el movimiento de hospicios de enfermos terminales, en particular el de St. Christopher en Londres, bajo el liderazgo de una enfermera, Cicely Saunders (Fox 1981). En contraposición con la tecnología médica y la cuasi invisibilidad del moribundo, el movimiento en torno a Saunders inaugura un debate moderno sobre las formas del 'buen morir'. St. Christopher se transformó en un lugar de peregrinaje de quienes buscaban una forma de morir que resistiera el poder de la sobremedicalización y ejerció también un fuerte influjo en Estados Unidos, en particular por políticas públicas interesadas en disminuir los gastos de salud en los últimos años y momentos de la vida (Seale 2003). Estos dos ejemplos son interesantes, más allá de sus aportes, porque se trata de mujeres del mundo médico, son las mujeres quienes tenían, ya sea en el hogar como en las instituciones médicas en tanto enfermeras, mayor responsabilidad en el cuidado y la interacción con los moribundos, y son ellas las que inician una postura crítica a las formas médicas y sociales de pensar, sentir y hacer frente a la muerte, presentando a su vez alternativas organizadas que encerraban una visión de la 'buena muerte' que intentaba contrapesar en alguna medida la creciente hegemonía médica (Fox 1981).

En cuanto a la sociología, los dos paradigmas centrales de la época, el funcionalismo y el interaccionismo simbólico, no serán ajenos al tema. El funcionalismo, en su intento de teoría social general, debe dar cuenta del tema, pero en un lugar marginal y de un modo más normativo que descriptivo o analítico. Esto se deriva de una primera constatación: la muerte en una sociedad de masas no es disfuncional, es un hecho normal y necesario para el equilibro homeostático de la especie. Influyó sin duda el modelo biológico de la sociología durkhemiana, para el cual la muerte individual era funcional para la continuidad del 'cuerpo social'. En consecuencia, al no ser un problema social, tampoco generó gran interés para esta escuela. Así, dentro de la orientación activa hacia el futuro como característica de las sociedades modernas, Parsons (1963) postulaba que la muerte sería menos temida y más aceptada que en el pasado. A fin de cuentas, se trata del final lógico de un camino de objetivos y logros, esperándose también que los deudos hagan su trabajo de duelo de forma rápida y eficiente (Riley 1983).

En el marco de la orientación activa hacia el futuro, se desarrolla un renovado interés desde la sociología económica por el estudio de los seguros. Influyente ha 
sido, sobre todo, el trabajo de Viviane Zelizer (1978). Tres son los interrogantes que el estudio de los seguros de vida permite develar. El primero se refiere a cómo se estima el valor de una vida; otros ubican los seguros en una línea histórica; ciertos autores lo entienden como otro resultado del proceso de secularización, ya que en un primer momento la Iglesia se opone a los seguros de vida, puesto que implicaban una forma de no aceptación del designio divino. Para otros, la extensión de los seguros es un indicador del declive de la vida comunitaria como red de protección. En todo caso, retomando la idea funcionalista de una orientación activa hacia el futuro, el lugar de los seguros y del seguro de vida en particular se transforma en un sugerente indicador sobre el lugar que la vida y la muerte ocupan en las sociedades modernas.

Si para el funcionalismo la muerte no sería un problema social en las sociedades modernas, por el contrario sí lo era en aquellas otras que Durkheim calificaba como 'simples', en las cuales prevalecía la solidaridad mecánica, y por lo cual la muerte causaba una gran disrupción, pues dejaba literalmente un lugar vacío en la organización comunitaria. Para antropólogos funcionalistas como R. Blauner (1966), su alto significado social era directamente proporcional a la envergadura de los ritos mortuorios (Palgi y Abramovitch 1984). En contraposición, en sociedades modernas complejas, unidas por el principio de solidaridad orgánica ligada a la división social del trabajo, la importancia social de la muerte individual disminuye, puesto que cada rol en la división social del trabajo es ocupado por muchos individuos y, por ello, los rituales públicos ante las pérdidas individuales pierden importancia, salvo en los casos de las grandes personalidades. Cobran mayor peso, en todo caso, las formas más privadas de duelo.

Pero fue el interaccionismo simbólico el que produjo la obra más importante del período en sociología: Awarennes of Dying, de B. Glaser y A. Strauss (1965). Se trata de un trabajo que marcó una época, influyó tanto a la sociología como a las profesiones médicas y se transformó en un clásico. El libro se basa en un trabajo comparativo en unidades de cuidados intensivos en hospitales. Tal como afirma Baszanger (1992), A. Strauss realiza una serie de operaciones importantes: busca las definiciones sociales de un fenómeno natural, preguntándose si las personas pueden estar muertas para las instituciones antes de estarlo realmente. Afirman que morir es un proceso lento, que implica un trabajo, e intentan comprender la gestión cotidiana de un hospital: cuando llega un enfermo en fase terminal, como el personal evalúa cuánto tiempo de vida le queda, qué cuidados vale la pena dispensarle, entre otros aspectos, condiciona su pasaje por la institución. El concepto central acuñado en Awarennes of Dying es 'contexto de conciencia'. Hace referencia a que cada actor sabe del estatus definido del enfermo según la institución y lo que los otros saben 
del enfermo y el enfermo mismo sabe constituyen los contextos de conciencia. Los autores definen cuatro contextos: conciencia cerrada (closed awareness), conciencia sospechosa (suspected awareness), mutuo engańo (mutual pretence) y conciencia abierta (open awareness). Cada tipo de conciencia implica comportamientos diferentes de las partes y sobre todo un trabajo dramático, con excepción de la última (conciencia abierta), para mantener esa definición de la situación (Baszanger 1992).

A su vez, cada contexto tiene ciertas ventajas y desventajas para el personal, los familiares y el enfermo. Cada contexto exige un tipo de comportamiento deseado del paciente, aun del moribundo. En la cultura anglosajona, por ejemplo, debe morir de un modo tranquilo, agradecido por los cuidados recibidos, despidiéndose de sus familiares. La negociación social llega hasta el último momento de la vida y parte de lo que se obtiene (como por ejemplo que no se le realicen más intervenciones médicas) estará relacionado con su adecuación a ese modelo virtuoso de un moribundo colaborador.

La otra obra capital, que proviene de la historia, es la de Philippe Ariès (1949, 1975, 1977, 1983). A diferencia de la mayoría de los trabajos sobre el tema, el autor no aísla ningún universal en relación a la muerte y se aboca a reconstruir y caracterizar la transición de los modelos de muerte en la sociedad europea occidental. Hay una relación, sostiene Ariès, entre los cambios en el sentimiento de familia y las actitudes ante la muerte. Esta, a su vez, está en relación con las variaciones en la conciencia de sí y del otro. Es precisamente esta interconexión entre los cambios en la idea de la familia y las actitudes ante la muerte la que le permite a Ariès trazar los hilos del proceso de individuación de la muerte en las sociedades europeas occidentales que es, creemos, la idea maestra del autor.

En 1941, Lucien Febvre había alertado sobre la ausencia de una historia sobre la muerte. Este vacío se modificó tardíamente, cuando la renovación historiográfica impulsada por la Escuela de Annales habilitó, y legitimó, nuevas preguntas y temas. El pasaje de la base económica a la superestructura cultural hizo posible un abanico más rico de temas dentro de los cuales la muerte encontró su lugar (Burke 1993, Revel 2005). El libro de Ariès (1977) irrumpe en este contexto y llena con originalidad un espacio vacante. Transita fluidamente por más de mil años de historia de Europa occidental y especialmente de Francia.

Esta perspectiva de muy larga duración, habitual en la historiografía, se elige también por una convicción: "los cambios del hombre ante la muerte son de por sí muy lentos, o se ubican entre largos períodos de inmovilidad. Los contemporáneos no perciben estos cambios, porque el tiempo que los separa supera al de varias generaciones y excede la capacidad de la memoria colectiva" (Ariès 1977:14) Las rupturas y las continuidades no son, sin embargo, recuperadas a partir de la 
serie y la cuantificación, sino a través de un análisis cualitativo sustentado en una diversidad notable de documentos: testamentos, novelas, fuentes litúrgicas y arqueológicas, iconografía. Los cambios son lentos y por momentos imperceptibles, $\mathrm{y}$ es en las clases dominantes en donde se manifiesta primero la innovación. Este modelo de difusión de arriba hacia abajo, muy criticado por sus colegas, atraviesa los cuatro modelos que articulan la reflexión: la muerte domesticada (hasta el siglo XII), la muerte propia (desde el siglo XII hasta fines del siglo XVIII), la muerte del otro (en el curso del siglo XIX hasta mediados del siglo XX), y la muerte invertida (en la época contemporánea). Se puede seguir, así, la implantación progresiva, desde fines de la Edad Media, del modelo de muerte cristiana que culminará en el Barroco hasta desintegrarse en el siglo XVIII, con el modelo de la muerte burguesa que se elabora desde las Luces al siglo XIX, para culminar en el presente con la muerte en el hospital.

No se trata sólo de la muerte; mejor aún, el interés se centra en sus estadios previos, en la agonía. Para Ariès, la forma de morir, cómo, dónde y con quiénes se llega a la muerte, es un dato vital para comprender históricamente las actitudes ante la muerte. En sus trabajos, pero también en el resto de los estudios comprendidos entre los años 1970 y 1990, la intención está en subrayar una actitud hacia la muerte en cada momento, poniendo el foco, preferentemente, en el punto de vista de los deudos y de las instituciones que administraban la muerte y desconociendo, por ende, los sentimientos y actitudes de un círculo más vasto de actores.

En la muerte domesticada el individuo sabía que iba a morir y cuando sentía la muerte próxima se preparaba para recibirla en la cama. La muerte era una ceremonia pública, sencilla en los ritos, organizada por el moribundo. A partir de los siglos XI-XII, en la muerte propia se produjo una reconciliación entre tres categorías de representación mental: la de la muerte, la del conocimiento de cada uno de su propia biografía y la del apego a las cosas que se poseyeron en vida. Al promediar la Edad Media, el hombre occidental rico, poderoso o letrado, se reconoce a sí mismo en su muerte: ha descubierto la muerte propia. A partir del siglo XVIII se tiende a dar un sentido nuevo a la muerte. La exalta, la dramatiza, pretende que sea impresionante y acaparadora. Pero el hombre no está tan preocupado por su propia muerte sino por la muerte del otro, el otro cuyo lamento y recuerdo inspiran en los siglos XIX y XX el nuevo culto de las tumbas y los cementerios.

A inicios del siglo XX, en América del Norte, Inglaterra y parte de Europa del Norte se perciben diferencias en los protocolos de los funerales, las costumbres del duelo y el rol del moribundo. Se tiende a evitar, no ya al moribundo sino a la sociedad y el entorno, el malestar y la emoción provocados por la agonía y la irrupción de la muerte en medio de la felicidad de la vida. Entre 1930 y 1950, la 
evolución va a precipitarse por el desplazamiento del lugar de la muerte, pues ya no se muere en medio de los suyos, sino en la soledad del hospital.

Estas ideas rectoras de una obra compleja y plagada de matices, brindan una interpretación sobre las actitudes adoptadas ante la muerte en el pasado y además participa de las discusiones en boga. En opinión de Ariès, las interdicciones sobre la muerte dominante en las décadas de 1960 y 1970 coincidían, al mismo tiempo, con su flagrante violación; es decir, con la irrupción de un discurso académico que, animado por sociólogos, psicólogos y antropólogos, reflexionaba sobre las experiencias de la muerte en el presente. El éxito rutilante de los trabajos de Ariès se comprende plenamente en este marco. L'Homme devant la mort ofrece un modelo seductor y útil sobre el pasado y especialmente muestra que ese pasado fue distinto y fue mejor. Oficia, en cierto modo, de prólogo de estos estudios y comulga también con una idea que subyace en ellos: la soledad del moribundo y la imposibilidad de hablar de la muerte en las sociedades modernas occidentales. Geoffrey Gorer, Ernest Becker Glasser y Strauss, Kluber-Ross y Lifton y Olson devienen esenciales.

En los años cincuenta, Gorer escribio el célebre artículo "The Pornography of Death" (1955 [1977]), en el cual, a partir de un estudio de 1.600 personas con experiencias cercanas de muerte, postula la idea del tabú de la muerte. El autor hace una comparación entre la era victoriana y la actualidad británica, y plantea que la muerte en el siglo XX ha venido a reemplazar al sexo en el lugar de los tabúes. Se la esconde, no se habla de ella, no se la nombra y cuando es imposible evitarla se alude a ella eufemísticamente. Es durante el duelo que la persona "tiene más necesidad de asistencia de la sociedad, como en la nińez y la adolescencia, y sin embargo es entonces cuando nuestra sociedad le retira su ayuda y le niega asistencia" (Gorer 1955:310). La sociedad aísla al muerto y al agonizante del flujo de la vida diaria, mucho más que en otros momentos de la historia.

Ernest Becker (1973), por su parte, señala el miedo a la muerte como central en la experiencia humana. En su obra se presentan dos interpretaciones contrapuestas que explican el origen de este temor central. Uno considera que el miedo a la muerte no es natural, sino que es creado por un tipo de socialización particular durante la cual los niños internalizan tal temor. La otra interpretación sostiene que el miedo a la muerte es el miedo básico del ser humano, detrás del cual están las fobias, la neurosis y demás temores. Becker se inclina por esta segunda postura, tal como Ariès, quien busca demostrar que el miedo a la muerte es una construcción cultural e histórica y que se origina en la modernidad.

Es este temor -terror diría Becker- el que estimula diversas formas de inmortalidad. En Symbolic Immortality, Jay y Olson (1974) postulan que una de las formas de hacer frente a la finitud de la vida, en particular a la idea de un vacío y de una 
desconexión con el resto de la humanidad a partir de la muerte, es mediante formas de inmortalidad simbólica. Se trata de cinco modos distintos: una inmortalidad biológica, mediante la continuidad en la familia, los hijos, pero también en comunidades más grandes como la nación, religión o clan (las llaman 'biosocial'). El segundo es de modo creativo, por medio del inventar, construir, escribir. En tercer lugar, una inmortalidad teológica, con un sentido más religioso clásico. En cuarto lugar, a través de ideas de continuidad con la naturaleza, tanto desde un punto de vista más religioso ("del polvo venimos y al polvo vamos") como en el ecologismo, mediante una comunidad con el mundo. Por último se refiere a una trascendencia experiencial mediante el amor, la danza, la contemplación artística.

En este segundo momento de 'explosión' de estudios sobre la muerte se genera un espacio de discusión interdisciplinario y metodológicamente heterogéneo. La concepción del tabú de la muerte y la soledad del moribundo en el hospital tendrán gran influjo y, sin ser las únicas ideas e incluso siendo contestadas por algunos análisis publicados en esos mismos ańos, gozaron y gozan de notable arraigo en el sentido común. Los trabajos se interesan mucho por las ideas, valores y creencias, $y$, desde una perspectiva durkheimiana y weberiana, por el sentido dado a la muerte y sus rituales. La perspectiva interaccionista y la mirada desde la historia permiten ver la muerte como un proceso co-coordinado y, al mismo tiempo, como un proceso temporalmente situado y sometido a cambios. Los ausentes para la sociología son el cuerpo; el muerto es más bien una idea, una ausencia. Los afectos tampoco ocupan un lugar central. Para los historiadores, en cambio, el cuerpo es un protagonista clave para detectar y comprender el proceso de individuación. Sin el cuerpo y sin su localización puntual por parte de los deudos, es difícil comprender e implementar el culto de las tumbas y los cementerios, así como el cultivo del recuerdo.

\section{Los años recientes: contextos e influencias teóricas}

Un tercer momento importante en la producción sobre el tema de la muerte se inicia en los años noventa del siglo pasado y continúa hasta el presente, con ciertos puntos de inflexión dentro del mismo. Las preguntas, una vez más, están fuertemente influidas por el contexto político y sociocultural. En primer lugar, en los países centrales se asiste a un claro retroceso de la pena de muerte, un incremento de la sensibilidad frente a la violencia política, una extensión de la prohibición de portar armas en Europa, una deslegitimación del asesinato por razones políticas y un proceso de deconstrucción del heroísmo militar (Memmi y Taïeb 2009). 
En una dirección contraria, al mismo tiempo, siempre desde la perspectiva de los países centrales, la muerte de los otros vuelve a escena, en dos formas: tanto víctimas como victimarios pueden ser englobados dentro de 'esos otros', como en los Balcanes, en las dictaduras del Cono Sur, en Ruanda y en otras matanzas y genocidios, pero también, luego del 11 de septiembre, los victimarios no son más ese 'otro' y se trata, entonces, de reflexionar sobre hechos que forman parte de ese 'nosotros' (en tales países) como el caso de tropas americanas y europeas, que matan en Irak y en Afganistán. Esto suscita críticas y reflexiones sobre, por ejemplo, el valor diferenciado de la vida y la muerte de las poblaciones para las potencias hegemónicas, según su nacionalidad y religión (Butler 2010).

Si el cáncer era la enfermedad central del período anterior, en este se agrega y cobra relevancia el HIV-sida. Será, sobre todo en sus inicios, una muerte fundamentalmente joven, con un proceso de morir del que se es consciente y que genera en gran medida la lucha política por el reconocimiento de los derechos de los enfermos y de ciertas minorías, en particular el movimiento gay, apoyados por gran cantidad de relatos biográficos, producción literaria y trabajos sociológicos.

En segundo lugar, la 'sociedad del riesgo', con Beck (1998) y Giddens (1994) como sus principales teóricos, ocupará un lugar central en el debate. Para Beck, en un mundo donde se ha generado una intensa división del trabajo, se diluye la responsabilidad sobre el riesgo, puesto que cada eventual responsable puede descargar su culpa en otras de las partes interdependientes. Sin embargo, si la responsabilidad sobre los riesgos puede en una escala diluirse, por otro lado, la modernidad tardía está sometida a la reflexividad, entendida como autoconfrontación frente a los resultados futuros de las propias elecciones. En consecuencia, habría también una mayor responsabilización individual por las consecuencias de las propias elecciones. Y, al mismo tiempo, una mayor responsabilidad por parte del estado.

Una segunda acepción es la idea de 'cultura de riesgo' de Giddens (1991). Esta se refiere a una creciente sensibilidad y a un cambio en las demandas políticas hacia la gestión de riesgos, incluyendo los ecológicos, de seguridad vial, alimenticia, urbana, terrorismo. En la cultura del riesgo, el estado también aparece como responsable de muertes por decisiones tomadas en temas de ecología, salud pública, seguridad, etc. A su vez, el estado es visto como responsable de las campañas de prevención, de información, de desarrollar vacunas contra pandemias, de dar información.

En los últimos años se ha sumado la discusión creciente en los países centrales de una mayor delegación en los individuos de la posibilidad de 'hacer morir': el derecho al suicidio, el derecho a la eutanasia. El poder se despliega también en el nacimiento, aborto, nacimiento de fetos de difícil sobre vida, contracepción, células madre, transplante, etc. A la par del desarrollo médico-técnico, que está en la base 
de estos debates, se trata de temas que tocan el centro de las concepciones éticas, filosóficas y jurídicas. Hay un trabajo legislativo y jurídico muy importante sobre el derecho a morir y en torno al comienzo de la vida, planteándose dilemas tales como, por ejemplo, el de la 'vida contra la vida' en el caso del aborto terapéutico (cuando se decide médicamente provocar un aborto porque la vida de la madre se considera en riesgo) en varios países de Europa (Weber et al. 2009).

Dos importantes libros, Seale (2003) y Howarth (2007) han realizado un análisis pormenorizado de los hallazgos de este último período y en gran medida las ideas que siguen se basan en ellos. De las influencias teóricas se destaca, por un lado, el conjunto de la obra de Foucault. La sociología médica recibe de este autor una mirada renovada sobre la forma en que el saber clasifica y gobierna, contribuyendo a adoptar una saludable mirada constructivista sobre el cuerpo, las disciplinas médicas y las enfermedades, así como de la división entre cuerpo y mente.

Pero también estará presente el Foucault de la gubernamentalidad. Recordemos con O’Malley (2006) que gubernamentalidad es un término que fusiona las ideas de gobierno y mentalidad para dar cuenta de las formas de administración del poder que surgen en la Europa del siglo XVII y que significaron un desplazamiento del mando simple y la obediencia hacia el aprovechamiento del autogobierno. La gubernamentalidad está ligada al desarrollo de ciertas disciplinas, como la economía y la estadística, o a conceptos como 'población', que tendrían una regulación autónoma, con leyes que los poderes no podían evitar y deberían tomar en consideración para el buen gobierno. La demografía, el intento de reducir las muertes, el control de las multitudes, la idea de la higiene pública como una necesidad de mantener distancias entre los cuerpos, para muchos autores cobran su sentido dentro de esta nueva técnica de gobierno.

Giddens es la otra influencia central. El giro reflexivo da lugar a la construcción de narrativas biográficas en el proceso de morir, a la vez que su noción de cultural scripts (guiones culturales distintos que pueden ser usados como recursos en diferentes construcciones biográficas) lleva a una revalorización del discurso médico como insumos que ayudan a dar sentido en el procesamiento biográfico de las enfermedades graves o terminales. El cuerpo recupera su centralidad. De forma distinta al cuerpo muerto que nunca dejó de ser estudiado por la antropología, un concepto central de esta época es el embodiment. De difícil traducción, una de las más aceptadas es la de 'en-carnación' (mantendremos el concepto en inglés). Esta hace referencia a la dimensión prediscursiva de la experiencia corporal. Según Macdonald et al. (2006), el embodiment se refiere a la presencia biológica y física de nuestros cuerpos, precondición necesaria para la subjetividad, emoción, lenguaje, pensamiento e interacción. De este modo, el cuerpo sentido, habitado, 
subjetivamente encarnado, será un elemento distintivo de este nuevo período; un cuerpo bastante ausente en la sociología del período anterior.

\section{Las principales preguntas}

Como mencionamos, la centralidad del HIV-sida conjugado con una preocupación por el embodiment interpela sobre la relación entre tipos de enfermedad y el proceso de morir. El tipo de enfermedad afecta de tal modo el cuerpo que la subjetividad es indisociable de ese embodiment (Seale 2003). Dentro de esta línea hubo intentos de articular el embodiment con la cultura. En efecto, la idea de 'culturas afectivas' implica también que las reglas del sentir y las reglas de expresión de las emociones incluyen el dolor (Harré 1986). Lo central, sin embargo, es la relación entre el sentido social de una determinada enfermedad y el padecimiento individual. Esto es, la concepción imperante sobre cada enfermedad o evento tiene consecuencias sobre la forma de sentirlo. Garro (1990) sostiene que el dolor por el cáncer es peor en las culturas donde se lo usa como metáfora del mal, como indicó Sontag, o con interpretaciones de vulgarización psicológica donde la enfermedad aparece como la emergencia incontrolada de lo reprimido, que además del dolor genera una cierta culpa en quien lo sufre. Lo contrario sucede con el dolor del parto, considerado un buen dolor, una experiencia vital en la transformación en madre. Así, no hay sólo buena o mala muerte, sino también un buen o mal dolor.

Una segunda línea de debate, presente en el período anterior, pero que se profundiza, es la relación entre estructura social, estilo de vida y mortalidad. Quizás los estudios más importantes que se realizan en las dos últimas décadas conciernen al impacto de variables sociales en las muertes, facilitados por el desarrollo estadístico. La muerte se objetiva como variable dependiente de una serie de otros hechos, transformados en variables independientes. Quizás los trabajos más importantes, en momentos de recrudecimiento del desempleo en Europa, fueron aquellos sobre el impacto del desempleo en la mortalidad. Estudios sobre los censos ingleses demostraron una mayor mortalidad, pero eso llevó a un estudio sobre la trayectoria social de los desempleados. Así, se pasó desde considerar el desempleo no sólo como causa de mortalidad y por ende de ser estudiado, a atenderlo en tanto indicador de una trayectoria laboral signada por la precariedad y por ende con una acumulación de elementos patógenos por malas condiciones laborales, estrés, de la que la desocupación será una de sus manifestaciones (Kessler 1999).

Luego de años y de una cantidad considerable de estudios que intentan mostrar que es el estilo de vida (alimentación, ejercicios, controles preventivos, estrés, 
etc.) lo que influye en las tasas de mortalidad más que la clase social, los trabajos terminan mostrando una gran fungibilidad entre estilos de vida y disposiciones materiales. Finalmente, vuelven a mostrar que el estilo de vida es una mediación en la relación entre clase social y mortalidad: los estilos de vida más saludables se encuentran en los sectores más favorecidos (Howarth 2007). También la relación entre mortalidad y género está atravesada por la situación social y la cultura. La medicina ha demostrado que genéticamente las mujeres están 'programadas' para vivir más que los hombres. Sin embargo, en algunos países muy subdesarrollados ellas viven menos debido a las malas condiciones de los partos y la falta de controles básicos de enfermedades ginecológicas, a lo que se suma una situación de dominación que dificulta el cuidado básico (Seale 2003).

La medicalización recibe ahora una mirada más matizada. La renovada mirada de Foucault (2004) sobre la medicina, además de otros aportes, dinamizó una historia cultural de la disciplina. Se realiza, así, una genealogía que muestra cómo la centralidad creciente del saber médico conllevó -y en cierta medida, fue gracias a - un rol central en la gestión de la vida y la muerte. La profesión médica recibió, procesó y con diversos grados se reconfiguró por las críticas recibidas en las décadas pasadas. En parte por ello, sobre todo desde las miradas más giddensianas, se rescata el lugar del discurso médico como un 'guión cultural' que permite dar sentido a la trayectoria biográfica ligada a la enfermedad (Seale 2003). En efecto, un paciente puede saber en qué estadio de una enfermedad se encuentra, los pasos siguientes, los tratamientos existentes, de acuerdo a lo cual organiza parte de su cotidianeidad; ello, asimismo, le proporciona recursos para dar sentido a la ruptura biográfica, ya señalada por Strauss décadas antes, como implica la enfermedad. La medicina provee a menudo una suerte de 'comunidad imaginada' conformada por aquellos que sufren un mismo tipo de enfermedad particular o la humanidad en un sentido general. La idea de humanidad se inviste de un sentido particular, es el marco en el cual se legitima la donación anónima de órganos o dando algún vestigio de sentido a los esfuerzos y los sufrimientos en los procesos médicos; como una contribución personal al avance de la medicina para el bien futuro de la humanidad. Una referencia a esa comunidad, la humanidad, está presente también en los bancos de células madres, de donantes de médula espinal transnacionales, entre otros. La no naturalidad de la humanidad (al menos su mayor preeminencia en términos médicos que políticos o religiosos, tales como existían ya mucho antes) se hace evidente en la comparación transcultural, así como en la resistencia japonesa al transplante por no concebir la reciprocidad anónima y generalizada que da cuenta de la idea de humanidad (Ohnuki-Tierney 1984).

La muerte, en todo este pasaje, también conoce reconfiguraciones. El cambio principal, si nos remontamos al trabajo de Glaser y Strauss y las diferentes conciencias, 
es la creciente preeminencia del 'derecho a saber'. En efecto, de ocultar la verdad al paciente y en última instancia negociar lo que sabe con sus familiares, se pasa a una alianza primera entre el médico y el paciente, tomando este último un rol de 'guardián' de la información que deja saber a sus allegados. Este nuevo acceso a la información también le permite, en caso de ser una enfermedad terminal, presidir su funeral, realizar los preparativos, arreglar sus cosas y hasta decidir la forma en que será su sepelio. No obstante, las diferencias interculturales se mantienen. Este desarrollo, si no único, es muy pronunciado en los países anglosajones y/o protestantes. Distinto es lo que pasa en los países latinos y en los orientales. En algunos se mantiene el silencio, en otros la preparación de la muerte es muy íntima y en forma religiosa; en cualquier caso, tomar en cuenta las diferencias interculturales es central.

Otros temas tratados en la fase pasada también se modifican. Por un lado se extiende el lugar y la importancia de los hospicios, y sobre todo cobran centralidad los cuidados paliativos. A nivel más conceptual, así como anteriormente se habían criticado por su carácter prescriptivo las fases del morir señaladas por Kubler-Ross, sucede algo parecido con las fases de conciencia de Glaser y Strauss. Aparecen como una forma demasiado normativa del morir, con un mayor peso a la resignación y se muestra que hay más resistencia, rabia, agresión respecto de lo que mostraban los primeros estudios. Posiblemente, el cambio en la relación médico-paciente también ha influido en las formas en que las emociones ligadas a la muerte pueden expresarse. En esta línea cobran centralidad las discusiones más ético-jurídicas sobre la eutanasia. También aparece otra mirada sobre el proceso de enfermedad terminal o invalidantes: una paulatina caída o salida de la cultura, en particular al dejar de participar de ciertos ritos cotidianos, como la comensalidad o, más hondamente, las conversaciones (Seale 2003). Pero no sólo se sale de la cultura: nuevos estudios muestran cómo el dolor y la enfermedad se expresan como una suerte de extranjerización del propio cuerpo. Los discursos sobre la enfermedad así lo muestran: en el dolor uno siente que no es uno, que el cuerpo lo traiciona, que ha sido invadido por un temible extraño.

Una idea-faro de los estudios de los años sesenta y setenta, como lo fue el tabú de la muerte, es revisitado, en particular en el marco de la influencia de Giddens, en la sociología británica. Mellor y Schilling (1993) afirman que la muerte está públicamente ausente, pero en cambio está muy presente privadamente, a partir de rituales, duelos y otras formas de recuerdo. Los autores señalan que en la modernidad tardía la conciencia de muerte tiene una nueva dimensión. Ello se debe en primer lugar al creciente rol del reordenamiento de las narrativas biográficas en el proceso de construcción de la identidad del yo; en segundo lugar, a la creciente identificación del yo con el cuerpo; y, finalmente, a la desacralización creciente 
de esferas de la vida social -la muerte entre ellas-, a partir de la secularización weberiana. Por ende, hay una serie de recursos para la reconstrucción narrativa del yo: terapias, libros, etc. El problema es que esa reconstrucción tiene como centro el cuerpo o el cuerpo que contiene el yo. En esto la muerte es más disruptiva y la conciencia de la finitud del yo se va viendo en el cuerpo. Así, disminuidas las posibilidades de una construcción social de la muerte individual, esta se hace más disruptiva en la modernidad tardía que en el pasado, y dar algún sentido en soledad a la finitud aparece como una tarea casi imposible.

Walter et al. (1995) discuten la idea de una muerte públicamente ausente. Afirman que la muerte está muy presente en los medios y por eso es muy intensa la idea de muerte pública. Los medios muestran cómo procesar la muerte, generan rituales de ruptura y reconstrucción, sobre todo ante catástrofes o muertes públicas de hombres ordinarios. Coinciden con este argumento, aunque de otro modo, los trabajos que hablan de las profesiones ligadas a la muerte; estos señalan cómo hay una mayor presencia pública (en esta escala) que deja en manos profesionales este pasaje. Finalmente, Seale (2003) afirma que en la vida cotidiana hay una necesidad psicológica de negar la muerte (para el psicoanálisis la muerte propia es impensable), hecho primordial para poder orientar la vida hacia objetivos más vitales.

Otro tema revisado es la relación de los vivos con los muertos. Se ha cuestionado la idea normativa de un duelo como un pasaje obligado, ni muy corto ni muy largo, que debía pasarse; el no hacerlo era negación, y el que durara mucho tiempo implicaba melancolía. Las terapias de duelo en el caso anglosajón eran una muestra de esta forma normativa que adoptó esa relación. Frente a esto se pasó a una mirada más abierta sobre tal relación, señalando la necesidad de una renegociación constante o periódica de los vivos con sus muertos (Howarth 2007). No hay necesariamente una etapa que se cierra, los muertos forman parte de la vida de los vivos y por eso van siendo objeto de distintas emociones, intensidades de recuerdo y formas de estar. En esta nueva concepción, el cuerpo no es el límite del self, sino que este perdura entre los vivos.

\section{A modo de balance}

En este recorrido historiográfico, la intención no fue cubrir todas las aproximaciones y a todos los autores, empresa imposible dada la cantidad impresionante de títulos, sino encontrar ciertos ejes temáticos y profundizar la mira, antes que dispersar, poniendo el foco en los aportes brindados por tres disciplinas: la antropología, la historia y la sociología. Al mismo tiempo fueron rescatados aquellos 
trabajos que, a nuestro juicio, propusieron una agenda de temas y perspectivas de investigación, innovaron metodológicamente o brindaron interpretaciones que alcanzaron gran impacto, ya sea por ser recurrentemente repetidas o por suscitar profundos debates al momento de ser publicadas o con posterioridad. Las líneas precedentes son, en definitiva, un punto de vista en sí mismo discutible sobre un tema que, nuevamente, despierta notable interés entre los estudiosos.

Como mostramos en este artículo, el estallido actual de los estudios sobre la muerte reconoce dos antecedentes, dos momentos, que no sólo lo preceden sino que además devinieron esenciales para la constitución de un campo de estudios. Más que retomar las especificidades de cada momento, y sin desconocer la particularidad de cada uno de los trabajos aquí referenciados, en estas líneas finales se tratará de recuperar ciertas persistencias, ejes analíticos y conceptuales que aparecen una y otra vez en los análisis. Si la muerte es un universal, también lo son ciertos nudos problemáticos tejidos en torno a ella. Desde los primeros trabajos abocados a comprender la proliferación de manifestaciones religiosas en las sociedades 'primitivas' y europeas, hasta en estudios más recientes que postulan un 'revival religioso' (Walter 1994), la relación entre muerte y creencias ha sido una constante y se ha convertido en un tópico. Es difícil sostener hoy la pérdida absoluta de referentes religiosos en las circunstancias y ceremonias que rodean la muerte e incluso la enfermedad. Es difícil sostener también hoy la sola presencia de las grandes religiones. Los historiadores han demostrado la complejidad del proceso de laicización y secularización, al mismo tiempo que han alertado sobre la necesidad de distinguir los usos y sentidos de manifestaciones y símbolos religiosos en el espacio público y en el privado. La secularización se define también a partir de la relación con y de la presencia de expresiones religiosas. Estas son múltiples y variadas en una sociedad dada, y también son heterogéneas para un individuo, así como pueden convivir además todas juntas en una misma ceremonia fúnebre. Es imposible, en definitiva, reflexionar sobre la muerte, más allá de la perspectiva analítica elegida, sin atender el conglomerado de creencias que la circunda y define. Como modeladoras de la experiencia social, son estas las que habilitan, en cierto modo, los distintos caminos o formas de inmortalidad que los trabajos han puesto en valor, apuntando a los discursos o reflexionando sobre las prácticas. Son también ellas las que explican en parte la estructura de los rituales. También aquí hay un continuum. Se ha escrito muchísimo desde las primeras reflexiones de Hertz y Van Gennep, y hemos aprendido las diferencias que pueden existir a través del tiempo y entre las distintas culturas. Configurados de maneras muy diversas, siguen siendo, no obstante, vitales. Para el muerto, los deudos y un círculo más vasto de individuos. Más que subrayar la pérdida de 
densidad o incluso la desaparición en la esfera pública de los ritos mortuorios, la apuesta está en recuperar sus sentidos -individuales y colectivos- que tuvieron y tienen. Siempre los individuos han preferido morir de una forma y no de otra. La calificación de buena muerte-mala muerte atraviesa, explícita o implícitamente, la inmensa mayoría de los trabajos. Sabemos que esta calificación varía con el tiempo y está históricamente situada. Una idea, bastante generalizada, sostiene que en el presente la buena muerte es aquella que sucede al final de una vida, en la vejez, por una enfermedad controlada por el saber médico que no implica una larga agonía. Otros, desde la perspectiva de la posmodernidad, postulan que la buena muerte es la que uno mismo elige.

Este puñado de ideas rectoras se incubó en los países centrales de Occidente. Aproximaciones descentradas y desde el Sur, por ejemplo, pueden sugerir nuevas ideas y matices interesantes. Quizás la más evidente sea la necesidad de romper con los esquemas binarios y en singular: religioso/laico; buena muerte/mala muerte; visibilidad/negación, etc. Y evitar generalizaciones indiferentes a la edad, el género, la clase y la forma de morir es central. Las altas tasas de mortalidad juvenil violenta entre las clases populares, especialmente masculina, de Argentina y la mayoría de los países latinoamericanos, abre perspectivas interesantes de análisis: por un lado relativiza el papel de la edad avanzada como variable sustantiva en la definición de una buena muerte y sitúa en primer plano la forma de morir (por abuso de drogas, muerte provocada por la policía, por arreglo de cuentas entre bandas, etc.); por el otro lado, la muerte como un hecho cotidiano, normal y en cierto modo esperable, permitiría volver a pensar 'viejos' temas como el de la relación entre estructura sociodemográfica y significados de la muerte, y la relación entre hecho cotidiano y naturalización. Finalmente, la precarización de las últimas décadas, el incremento de la pobreza y la relación simple que suele establecerse entre esta y el aumento de la criminalidad y la inseguridad, reactualizan la muerte como posibilidad para distintos actores: para las clases populares, porque ellas tienen que gestionar cotidianamente con la muerte; para las otras clases sociales, porque ven en la inseguridad y en la sensación de inseguridad que denuncian, la posibilidad concreta de su muerte. 


\section{Referencias bibliográficas}

Ariès, Philippe, 1949. Attitudes devant la vie et devant la mort du XVII au XIX'e siècle, quelques aspects de leurs variations. Paris: INED.

1975. Essais sur l'histoire de la mort en Occident: du Moyen Âge à nos jours. Paris: Seuil. , 1977. L'Homme devant la mort. Paris: Seuil. 1983. Images de l'homme devant la mort. Paris: Seuil.

Barrán, José Pedro, 1991. Historia de la sensibilidad en Uruguay, Tomo I: La cultura "bárbara” (1800-1860). Tomo II: El disciplinamiento (1860-1920). Montevideo: Ediciones de la Banda Oriental.

Baszanger, Isabelle, 1992. "Les chantiers d'un interactionniste." En Isabelle Baszanger, comp. La trame de la negotiation. Paris: L'Harmmatan.

Beck, Ulrich, 1998. La sociedad del riesgo. Hacia una nueva modernidad. Buenos Aires: Paidós. Becker, Ernest, 1973. The Denial of Death. New York: The Free Press.

Blauner, Robert, 1966. "Death and Social Structure." Psychiatry, № 29, 378-394.

Burke, Peter, 1993. La revolución historiográfica francesa. La Escuela de los Annales: 1929-1989. Barcelona: Gedisa.

Butler, Judith, 2010. Marcos de guerra. Vidas lloradas. Buenos Aires: Paidós.

Faunce, William, y Robert Fulton, 1957. “The Sociology of Death: a Neglected Area or Research.” Social Forces, N³6, 205-209.

Foucault, Michel, 2004 [1963]. El nacimiento de la clínica. Arqueología de la mirada médica. Madrid: Siglo XXI.

Fox, Renée, 1981. "The Sting of Death in American Society." Social Service Review, Vol. 5, $\mathrm{N}^{\circ} 1,42-58$.

Garro, Linda, 1990. "Culture, Pain and Cancer." Journal of Palliative Care, Vol. 11, № 4, 34-44.

Giddens, Antonhy, 1991. Modernidad e identidad del yo. Madrid: Península. 1994. Consecuencias de la modernidad. Madrid: Alianza.

Glaser, Bernard, y Anselm Strauss, 1965. Awareness of Dying. Chicago: Aldine.

Gorer, Geoffrey, 1977. Death, Grief and Mourning in Contemporary Britain. London: Arno Press. Habenstein, Robert, y William Lamers, 1955. The History of American Funeral Directing. Milwaukee, Wis.: Bulfin Printers.

Harré, Rom, 1986. The Social Constructions of Emotions. Oxford: Blackwell.

Hertz, Robert, 1960 [1907]. Death and the Right Hand. Rodney Needham y Claudia Needham, trads. London: Cohen and West.

Howarth, Glennys, 2007. Death and Dying. A Sociological Introduction. Cambridge: Polity Press. Jay, Robert, y Olson, Eric, 1974. "Symbolic immortality". En Living and Dying, London: Wildwood House. 
Kessler, Gabriel, 1999. "El impacto social del desempleo. Aportes de la experiencia internacional." Socialis, $\mathrm{N}^{\circ}$ 1, 93-115.

Kübler-Ross, Elizabeth, 1969. On Death and Dying. New York: McMillan.

Lifton, Jay, y Olson, Eric, 1974. Living and Dying. New York: Praeger.

Lomnitz, Claudio, 2006. Idea de la muerte en México. México D.F.: Fondo de Cultura Económica.

Macdonald, Raymond, David Hargreaves y Dorothy Miell, eds., 2002. Musical Identities. Oxford: Oxford University Press.

Malinowski, Bronislaw, 1948. Magia, ciencia y religión. Madrid: Planeta Agostini.

Mellor, Philip, y Chris Shilling, 1993. "Modernity, Self-identity and the Sequestration of Death." Sociology, Vol. 27, №3, 411-431.

Memmi, Dominique, y Emmanuel Taïeb, 2009. "Introduction. L'État et la mort." Sociétés Contemporaines, $\mathrm{N}^{\circ} 75,5-16$.

Mitford, Jessica, 1963. The American Way of Death. New York: Simon \& Schuster.

O’Malley, Pat, 2006. Riesgo, neoliberalismo y justicia penal. Buenos Aires: Ad-Hoc.

Ohnuki-Tierney, Emiko, 1984. Illnes and Culture in Contemporany Japan: an Anthropological View. Cambridge: Cambridge University Press.

Palgi, Phyllis, y Henry Abramovitch, 1984. "Death: A Cross-Cultural Perspective." Annual Review of Anthropology, Vol. 13, 385-417.

Parsons, Talcott, 1963. "Death in American Society: A Brief Working Paper." American Behaviour Sciences, N 6, 61-65.

Radcliffe-Brown, Alfred, 1972 [1952]. Estructura y función en la sociedad primitiva. Barcelona: Península.

Revel, Jacques, 2005. "Historia y Ciencias Sociales: los paradigmas de los Annales." Un momento historiográfico. Trece ensayos de historia social. Buenos Aires: Manantial.

Riley, John, 1983. "Dying and the Meanings of Death.” Sociological Inquiries, Annual Review of Sociology, Vol. 9, 191-216.

Robben, Antonius, 2004. "Death and Anthropology: An Introduction.” En A. Robben, ed. Death, Mourning and burial. A Cross Cultural Reader. Cornwall: Blackwell.

Seale, Clive, 2003. Constructing Death. The Sociology of Dying and Bereavement. Cambridge: Cambridge University Press.

Sontag, Susan, 1977. Illness as Metaphor. New York: Farrar, Straus \& Giroux.

Stouffer, Samuel, Edward A. Suchmann, Leland C. de Vinney, Shirley A. Star y Robin M. Williams, Jr., 1949. The American Soldier. Princeton: Princeton University Press.

Sudnow, David, 1967. Passing On: The Social Organization of Dying. Englewood Cliffs, NJ.: Prentice-Hall.

Van Gennep, Arnold, 1960. The Rites of Passage. A Classic Study of Cultural celebrations. Chicago: The University of Chicago Press. 
Venbrux, Eric, 2007. "Robert Hertz's seminal essay and mortuary rites in the Pacific region, Journal de la Société des Océanistes 124. N 1, 5-9.

Vovelle, Michel, 1970. Vision de la mort et de l'au-delà en provence d'après les autels des âmes du Purgatoire. Paris: Colin.

, 1973. Piété baroque et déchristianitation. Paris: Plon.

, 1974. Mourir autrefois. Attitudes collectives devant la mort aux XVII et XVIII ${ }^{\text {eme }}$ siècles.

Paris: Gallimard.

, 1982. Ideologías y mentalidades. Barcelona: Ariel.

1983. La mort et l'Occident de 1300 à nos jours. Paris: Gallimard.

Walter, Tony, 1994. The Revival of Death. London: Routledge.

Walter, Tony, Jane Littlewood y Michael Pickering, 1995. "Death in the News: the Public Invigilation of Private Emotion.” Sociology, Vol. 29, No 4, 579-596.

Weber, Jean-Christophe, Dominique Memmi, Tierry Rusterholtz y Catherine Allamel-Raffin, 2009. "Administrer le foeticide." Sociétés Contemporaines, $\mathrm{N}^{\circ} 75,17-35$.

Zelizer, Viviana, 1978. "Human Values and the Market: the Case of Life Insurance and Death on 19th-Century America." American Journal of Sociology, Vol. 84, № 3, 591-610. 